\title{
The role of microvesicles on immune function in response to cancer
}

\section{Ritu Jaiswal*1, Deep Pokharel ${ }^{1}$ and Mary Bebawy ${ }^{1}$}

Discipline of Pharmacy, Graduate School of Health, University of Technology Sydney, NSW 2007 Australia

Cell to cell communication is vital for the co-ordination of physiological process and the regulation of an organism's phenotype. More recently communication via extracellular membrane vesicles has gained recognition. We first described a novel mechanism for the spread and dominance of multidrug resistance (MDR) and enhanced metastatic capacity in cancer via submicron microparticles (MPs). MPs are plasma membrane vesicles released spontaneously from various cell types, carrying bioactive material and are implicated in different physiological and pathophysiological processes. Through this communication apparatus, cancer cells can acquire and secure a survival advantage by various mechanisms. This study aims to examine a role of MPs in altering immune cell function in cancer.

The effects of MPs isolated from human breast cancer cells were examined on antigen presenting cells (APC) in vitro. MP-mediated effects on cell phenotype and functionality was assessed by cytokine profiling and migration assay. We observed a cancer cell induced change in immune cell phenotype and functionality which have the potential to support a reduced global immune response in cancer. The elucidation of this pathway provides novel therapeutic strategies which can be exploited for the treatment of cancer.

\footnotetext{
*Presenting author
} 